\title{
Impact of Deep Learning in Medical Imaging: a Systematic New Proposed Model
}

\author{
Surayya Ado Bala, Shri Kant, Khemendra Kumar
}

\begin{abstract}
For years' radiologist and clinician continues to employs various approaches, machine learning algorithms included to detect, diagnose, and prevent diseases using medical imaging. Recent advances in deep learning made medical imaging analysis and processing an active research area, various algorithms for segmentation, detection, and classification have been proposed. In this survey, we describe the trends of deep learning algorithms use in medical imaging, their architecture, hardware, and software used are all discussed. We concluded with the proposed model for brain lesion segmentation and classification using Magnetic Resonance Images (MRI).
\end{abstract}

Index Terms: Deep learning architectures, data augmentation machine learning, medical imaging

\section{INTRODUCTION}

Deep learning (DL) has touched almost every domain and day to day activities over the last decade. It is a new machine learning paradigm that focuses on learning with deep hierarchical models of data. DL is a kind of Artificial Neural Network (ANN) that comes into existence in the '80s [1] and become apparent in 2006 [2]. In 2012, a deep learning-based architecture called Convolutional Neural Network (CNN) was used by researchers for ImageNet classification where they won a great success in a computer vision challenge. Since then, many researchers adapted DL in medical image analysis and other computer vision task. Advancement of technology, self-learning [3], improvement of existing learning methods , and development of computing capabilities, especially graphics processing unit (GPUs) whose serve as the fundamental factors to the success of DL. These factors led to the development of well successful models used for medical image analysis which shows inherent ability to diagnose disease, because of its ability to segment and classify diseases which produced superb results as highlighted in an editorial of DL techniques [4].

Medical Imaging is an essential part of the initial detection, diagnosis, treatment, and prevention of disease. Medical images such as X-ray, positron emission tomography (PET), ultrasound, and magnetic resonance images (MRI) are used for the precise diagnosis of disease. The analysis is based on image acquisition and image interpretation.

Revised Manuscript Received on October25, 2019

Surayya Ado Bala, Department of Computer Science and Engineering Sharda University, Greater Noida, India.

Shri Kant, Department of Computer Science and Engineering Sharda University, Greater Noida, India.

Khemendra Kumar, Department of Radio Diagnosis, Sharda Hospital, Greater Noida, India.
Image acquisition is the initial stage in medical image analysis for acquiring images, which was significantly improved with the advancement of devices, that received the data at a faster rate with more increased in resolution. Radiologists made interpretation of medical images which is very challenging, time-consuming, and also having disparity across interpreters [4].

Recently, researchers in the medical imaging field have focused on bringing new techniques. Before the existence of these techniques, visual inspection of medical images is done by trained and experienced radiologists, computer algorithms using traditional machine learning, image processing, and pattern recognition [5]. All these methods are not able to recognize the problem uniquely, and there are variations in the diagnostic. DL tackle this problem by identifying the sophisticated features of the particular region so that radiologist can make the right decision for the treatment.

In this paper, we explore the impact of DL in medical imaging and discuss why DL is preferred over the traditional machine learning in section II. We describe the hardware and software platforms used for DL in section III. The details of DL architectures are presented in Section IV, while the application of DL in medical imaging was discussed in section V. In section VI, we present a proposed model for segmentation, detection, classification and, conclude in Section VII.

\section{WHY DEEP LEARNING OVER TRADITIONAL MACHINE LEARNING}

Deep Learning is impressive not only because its level of performance is higher, but can build a deeper not shallow network with millions of neurons and hundreds of layers . This gives DL an edge over machine learning, which requires feature engineering, as shown in Fig.1. Feature engineering requires domain expertise, and it is a complicated task which consumes a lot of time .

The features need to be handcrafted in traditional machine learning that is extracted then selected while in DL it is done automatically.

Traditional machine learning is a vigorous algorithm for identifying patterns or image features that can be applied to medical images for the prediction, classification, or diagnosis of interest using selected features [6] [7]. 


\section{Impact of Deep Learning in Medical Imaging: a Systematic New Proposed Model}

These features were used as input to the traditional machine learning system, which also tends to select useless or redundant features, also not including the useful features required [6].
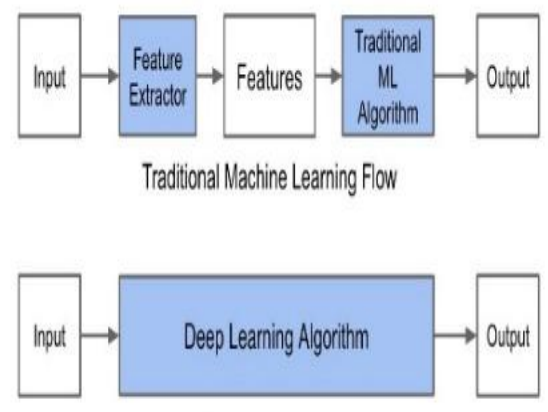

Deep Learning Flow

Fig. 1: Traditional Machine Learning Vs. Deep Learning

\section{H/W AND S/W PLATFORMS USED FOR DEEP LEARNING}

Hardware and software play key roles in the development and application of deep learning.

\section{A. Hardware}

Graphics Processing Unit (GPU) is one of the critical factors responsible for the advancement and success of DL. It provides higher performance and a potent processing unit for computing applications [8] with its computing platforms that is Compute Unified Device Architecture (CUDA) and Open Computing Language (OpenCL) [9]. GPU is a powerful computing machine that performed mathematical operations with high performance than central processing units (CPUs) with more execution threads [10].

\section{B. Software}

In DL there are varieties of open-source software frameworks used which includes;

\section{i. Convolutional Architecture for Fast Feature Embedding (Caffe)}

Caffe is an open-source software created and developed by the Berkeley Vision and Learning Centre (BVLC) [11]. It explores different architecture and implements them efficiently for learning and prediction. Matlab, C, C+, and python are interfaces that support Caffe.

\section{ii. Theano}

Theano is an open-source python library developed in Montreal by members of Montreal Institute for Learning Algorithms (MILA) lab. It is used for optimizing and evaluating mathematical expressions with multi-dimensional array [12]. It was designed to perform the computation for a vast neural network and to increase development and execution time for the machine learning algorithm [13].

\section{iii. Tensor Flow}

Tensor Flow is a DL framework for high computation performance created by google brain team of researchers and engineers [14] using data flows for machine learning and deep neural networks research. It supports $\mathrm{C}++$ and Python interfaces and runs on both GPUs and CPUs.

\section{iv. Torch}

Torch [15] is an efficient scientific computing framework, very easy to use, and provide broad support for machine learning. It Provides a Lua interface which is a supremely lightweight scripting language used by Facebook Artificial Intelligence research [16]

\section{DEEP LEARNING ARCHITECTURES}

\section{A. Supervised Learning}

Supervised learning is the process whereby for every input variables $\mathrm{X}$, there is a labeled output variable $\mathrm{Y}$, which are used to train the machine and get the desired outputs. There are several Supervised learning models which include;

\section{i. Neural Network}

Neural Network is learning algorithms that mimic the brain, identify the pattern, and form the basis of most DL algorithm [5]. It is made up of inputs, weights, activation function, and biases, as shown in Fig. 2 below. Neural networks training involves many stages or epochs when new data (input) is added. Delta rule is used as the learning process for the adjustment of the weights, training, and implementation by exploiting back propagation routine [17]. The network weights are initialized randomly and trained using an iterative training method called gradient descent. The gradient descent was also used for the optimization and minimization of the disparity that occurs in the network output, and the targeted network output.

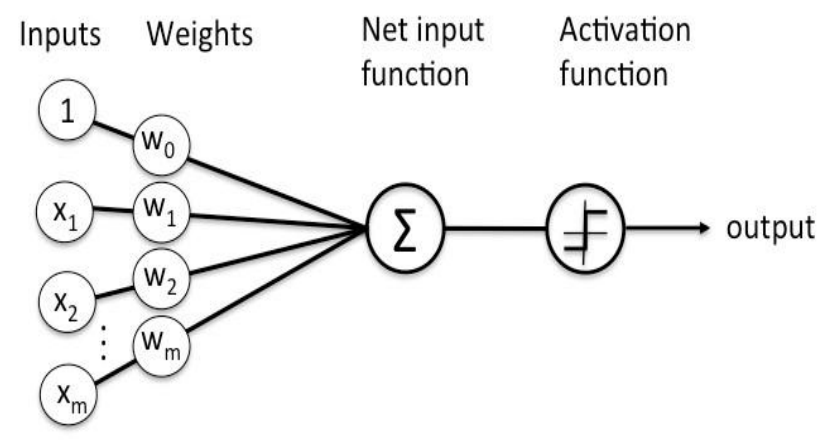

Fig. 2: Neural Network

\section{ii. Deep Neural Network}

The shallowness of ANN makes it fail with more than four hidden layers; this leads to the introduction of Deep Neural Network (DNN) [18-20]. The DNN architecture comprises input and output layers, with tens to hundreds of hidden layers. 
The amount of hidden layers and the model architecture depends on the application. The architecture used for the detection of breast cancer proposed by [21] is entirely different from the one proposed by [22] for the detection of breast cancer. In the DNN architecture information are passed from the input to the output layer through the hidden layer in a sequential manner called feedforward network. In each layer, various computation is performed before any output is generated, which become an input to the next layer.

DNN is feedforward networks that model complex non-linear correlation of data from the input to the output layer without looping back, as shown in Fig. 3. Training a deep neural network sometimes is complicated because if errors are backpropagated to the few first layers and the network considers the errors inconsequential which can be neglected, this leads to vanishing gradient descent problem. However, sophisticated variant backpropagation [23] have overcome vanishing gradient descent problem, but it slows down the learning process. Different approaches have been provided by DL to train DNN architecture. In training the DNN architecture, labeled data are provided in case of supervised learning to learn the weights in such a way that it minimized the errors between desire and the target output values for either classification or regression [17].

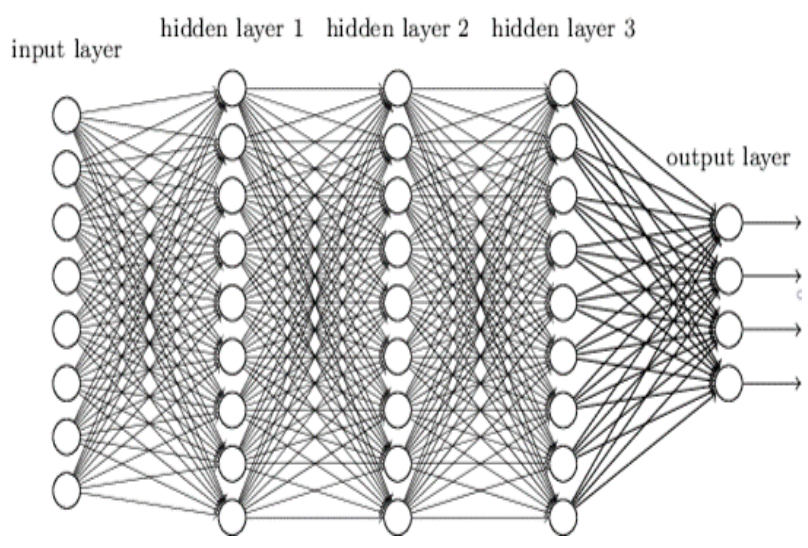

Fig. 3: Deep Neural Network

\section{i. Convolutional Neural Network}

Among the successful architecture of DNN is the CNN, mostly utilized in image analysis task. The popularity of $\mathrm{CNN}$ architecture is due to its application in computer vision and other variants of its kind [24]. It is considered as the most competent and accessible deep learning algorithm, especially for image analysis and recognition. It features selection capability of retaining the local image relation at the same time performing dimensionality reduction makes it suitable for any image analysis tasks [25]. Furthermore, it increased the computation efficiency of various models through the decrease of learnable parameters at the same time, capturing vital features needed to perform the classification task. CNN extract features using multiple filters which learn high-level features from the original data of an image then used for classification, as shown in Fig. 5. CNN architecture exhibit certain similarity with multi-layer perceptron (MLP) that is, the images are converted to pixels, perform some pre-processing on them then fed the raw pixels into the CNN [26]. It takes the raw image pixel as input, and transforms it via Convolutional Layer, Rectified Linear Unit [27] and Pooling Layers [28]. After various computations are performed on the raw pixels by the predecessor's layers, the result is pass to the fully connected layer. This layer shows the classification by assigning a class score to the image. Convolution is the process of performing manipulation on two mathematical functions. To analyze the images, they have to be converted to a pixel value, use as an input to the network model. Then the filter function is performed, as depicted in Fig. 4 . The RELU serves as the activation function, which changes all the negative values to zero. They speed up the computation of the model training and eliminate the vanishing gradient problem. The pooling layer is used to reduce the parameters numbers, which lies in between the convolution layer and the activation layer. As stated in [29], the most often layer used is max pooling as other layers exist. In max pooling, the number of layers is reduced by selecting the highest value. The output of the previous layers function as the input; then it used to calculate the probability score and classify them based on the result.

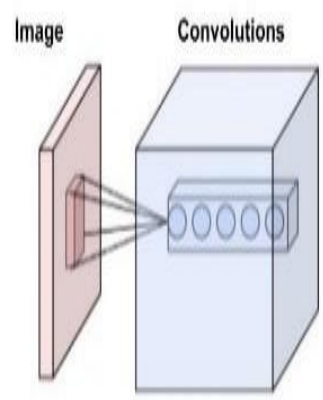

Fig. 4: Convolutional Layer

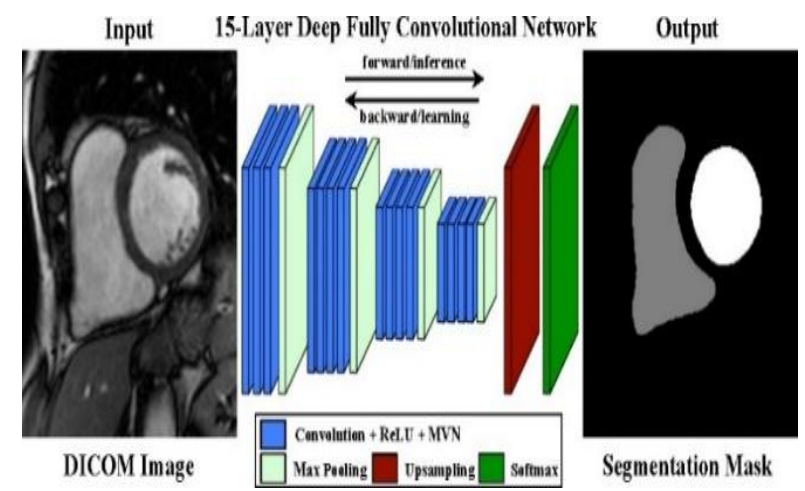

Fig. 5: Convolutional Neural Network for Segmentation 


\section{Impact of Deep Learning in Medical Imaging: a Systematic New Proposed Model}

\section{B. Unsupervised Learning}

In unsupervised learning, unlabeled data are used for learning. The data is used to describe the hidden structure. There are several unsupervised algorithms which include;

\section{i. Autoencoders}

Autoencoders are used for unsupervised of coding .The codings are the input data; they study the feature representation in an unsupervised way without labeled data. As illustrated in Fig. 6 , inputs are transformed into inputs and outputs with encoder and decoder in between them [30].

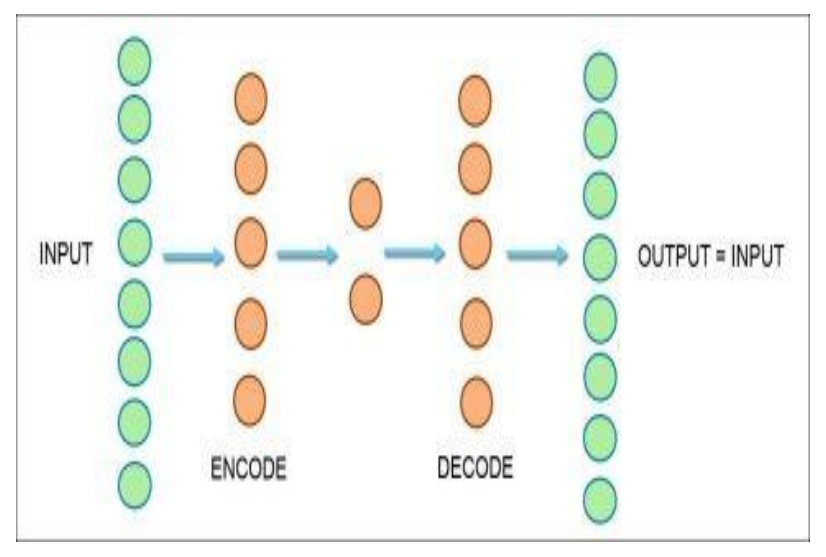

Fig. 6: Autoencoders

\section{a. Stacked Autoencoders}

They are a type of autoencoders in which separate training of different subset of encodes are done before they are stacked together [31].

\section{b. DE noising Autoencoder}

DE noising Autoencoder is another type of autoencoder where noise is added, usually Gaussian noise to the hidden layer [31] or through dropout, that is a random selection of some neurons in the first hidden layer and turning them off. This process forces the model to learn some useful coding in other to reconstruct back noise-free input at the output layer.

\section{c. Sparse Autoencoder}

Sparse Autoencoder is the type of autoencoders that learn essential features in the input data that can be useful at the output layer. The features extracted from data that are not important or useful are set to zero [32]. The process of sparse representation of input is essential in the initial training of the model for classification tasks. They imposed sparsity on the hidden layer during model training by increasing the number of hidden layers than the previous one.

\section{ii. Restricted Boltzmann Machines}

Restricted Boltzmann Machines (RBMs) are bidirectional graphical and generative models that consist of two unit layers (visible and hidden) [33], no direct connection between the hidden to hidden and visible to visible. The probability distribution from the initial input in $\mathrm{RBM}$ is estimated through the reconstruction of the original input using a backward pass learning procedure.

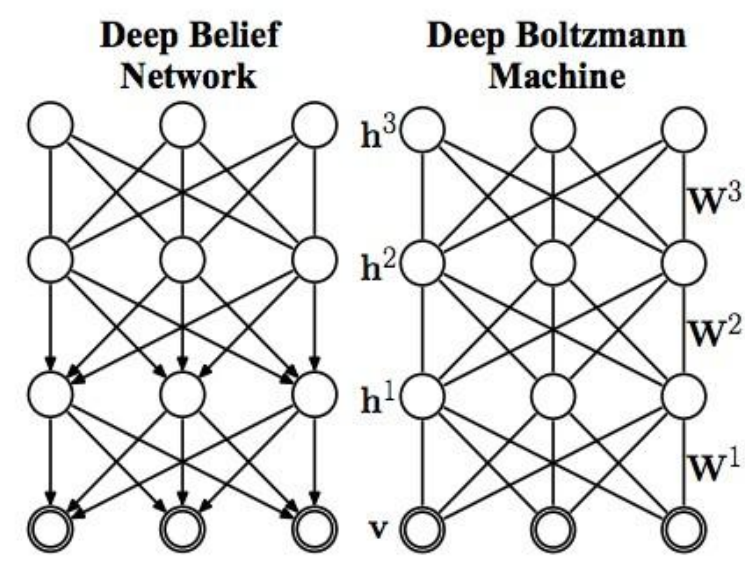

Fig. 7: Deep Belief Network and Deep Boltzmann Machine

\section{a. Deep Belief Network}

Deep Belief Network (DBN) was much used for the reawakening in deep learning [34]. DBN train the network in a layer by layer way [35], though it works hierarchically as the real-world data do, for which higher-level layers learn more top feature and lower ones also determines smaller features.

\section{b. Deep Boltzmann Machines}

It is a variant of neural network based on Boltzmann family [36]. DBM is trained in a layer by layer way as it is in DBN. As shown in Fig 7. Both DBN and DBM possess undirected connections. In DBN the time inference is very high as compare to DBN, which makes optimization very difficult while training the network [37].

\section{Semi-Supervised Learning}

Semi-supervised learning is the amalgamation of supervised and unsupervised learning. Generative Adversarial Networks is an example of Semi-supervised learning.

\section{i. Generative Adversarial Networks}

Generative Adversarial Network (GANS) are generative models used for image synthesis [38]. They generate useful information (data) with limited available training data . GANs, as shown in Fig 8., comprise two competing models, the generator and the discriminator, which are used simultaneously. The Generator $(\mathrm{G})$ generates fake data(image) from random noise that mimics the original dataset (image). The generated and original datasets(images) are feed into the discriminator. The discriminator, which is a conventional CNN, will differentiate between the original dataset(images)and the generated images by assigning probability label $(0,1)$.

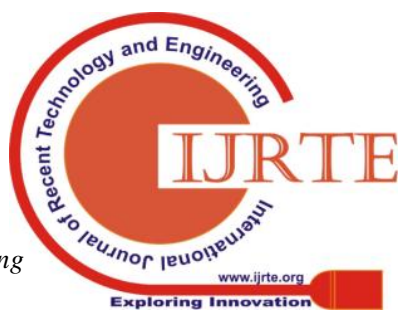


Zero(0) for the generated ( fake ) image and one (1) for the original image. The posterior probability is given by $\mathrm{P}($ Label/Data).

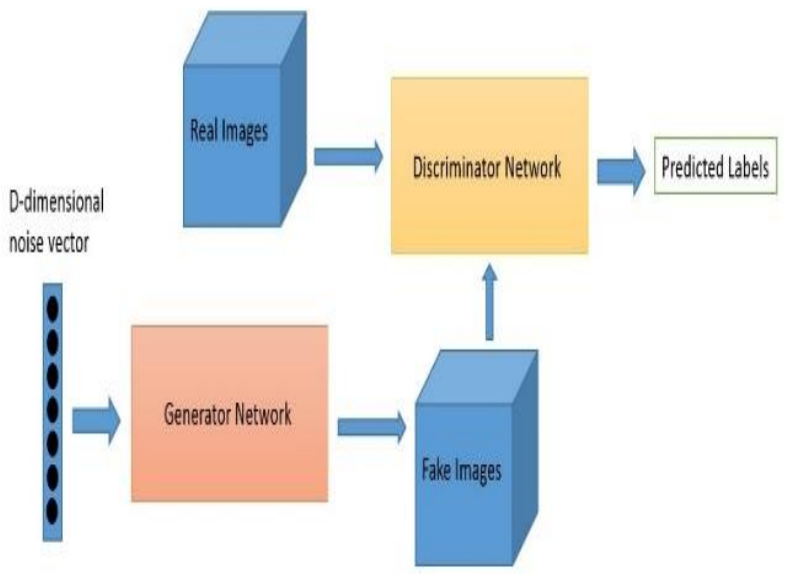

Fig. 8: Generative Adversarial Network

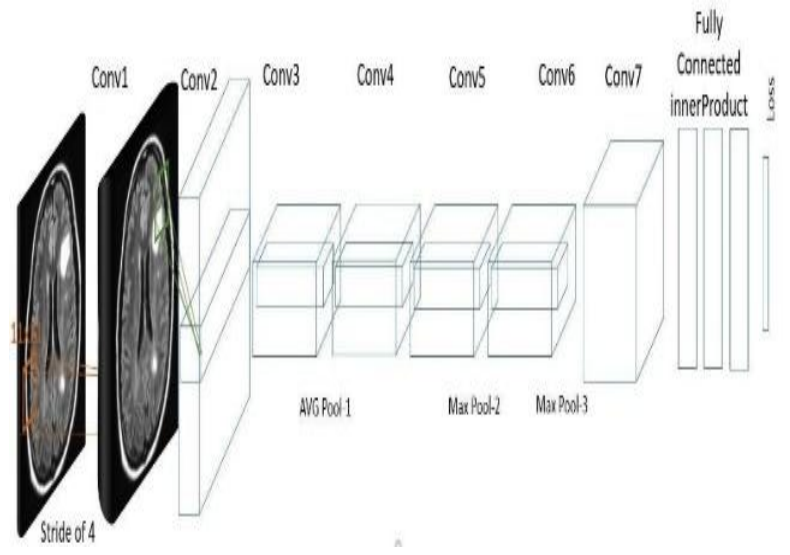

Fig. 9: Classification Architecture

\section{APPLICATION OF DEEP LEARNING IN MEDICAL IMAGING}

DL has momentous achievements in many domains, especially in medical image analysis. With the current advancement in DL and GPU, researchers in the field of medical image analysis have achieved excellent results in detection,segmentation,classification, and registration [39].

\section{A. Detection}

Detection of abnormality (Lesion) is a pre-processing task before segmentation in medical image analysis, for identification of disease in different modalities.

In the literature, there are various detection algorithms proposed, which include both generative and discriminative models. In [40] a brain lesion detection was done, an accuracy of $91 \%$ sensitivity, and $59 \%$ specificity was achieved using multi-sequence images that include $\mathrm{T} 1, \mathrm{~T} 2$, and flair with their annotated images. Also, approaches were proposed in [40-42]. In [41], supervised and unsupervised methods are amalgamated together to performed lesion detection on MRI images. Detection of myocardial abnormalities is carried out in [43] for cardiac MRI.

\section{B. Segmentation}

Segmentation is a method of apportioning images or data to multiple sub-regions manually, semi-automatically, or automatically based on pixels or essential features [44]. In recent studies, segmentation has been applied to medical images [45]. Organs, lesions, and other hidden parts segmentation of the medical images simplified the analysis of experimental parameters related to the shape and location. There are various DL architectures used by many researchers in segmenting different anatomical structures from different modalities. These architectures include 2D and 3D CNN architectures [46]. A deep learning-based model architecture was proposed in [29] for the segmentation of sclerosis lesion in MR images. The architecture contains a 3D-CNN, which is made up of two pathways, the convolution learned the hierarchical feature representation and a deconvolutional path that provide both the unpooling layer and the deconvolutional layer. In [47], a 3D DL architecture was proposed and used for the segmentation of brain tumor.

Also, in [49], a two-path architecture with eleven layers has been proposed, the architecture was based on Deconvolution Neural Network (DCNN) for brain lesion segmentation. They used dense training with 3D patches which utilized a 3D fully connected conditional random field.

\section{Classification}

In medical image analysis, classification is a vital task. It is used for classifying of diseases based on their features for further diagnosis.

Researchers suggested many DL models for classification of medical images in different anatomical and different modalities. In [48-50], new models have been proposed for classification. They achieved an accuracy from $85.5 \%$ above . The proposed network is trained from the extracted patches of the whole image, which serves as the input to the models.

\section{Registration}

Medical image registration is an image analysis task of computing. The coordinate transform the medical image from one form to another, with the input images, a deep learning algorithm is used by [51] [52] to guess the image registration using transform parameters.

\section{PROPOSED MODEL}

A deep learning model for brain lesion detection, segmentation, and classification using MR images to differentiate between Neurocyserticorsis and Tuberculoma is proposed as illustrated in Fig 10. 


\section{Impact of Deep Learning in Medical Imaging: a Systematic New Proposed Model \\ REFERENCES}

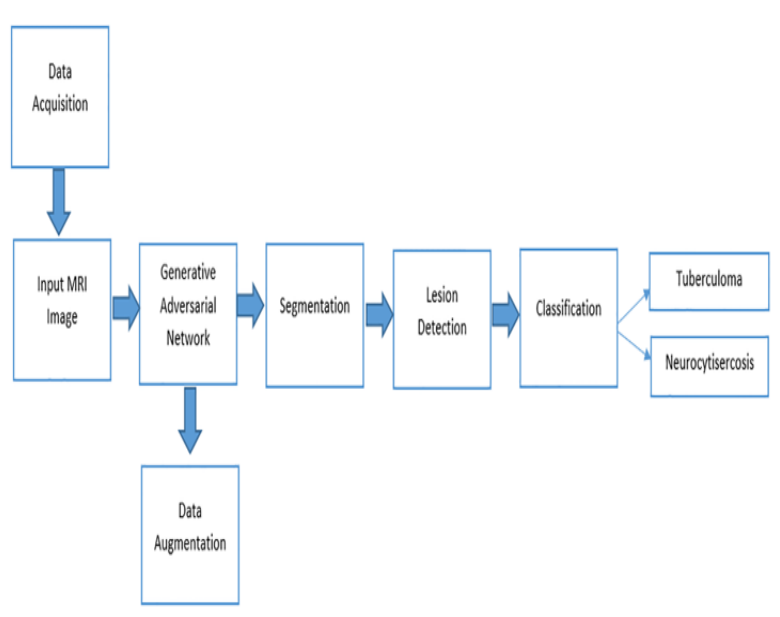

Fig. 10: Process for the Proposed Model

The model will be developed using CNN. GAN will be used for data augmentation. GAN works by training two different networks. Input image for the network will be three channels, and we will use axial, coronal, and sagittal planes in a Volume-of-Interest (VOI) for each category.

In the detection and the segmentation phase, we will use a $\mathrm{CNN}$ architecture, which will provide valuable information about size, location, and categories of the lesions as well.

For classification, the architecture will be based on the network proposed by [53] as shown in Fig.9.The network comprises of three pooling layers and seven convolutional layers. The learned features from the $\mathrm{CNN}$ are convolved to the input data which uses $3 \mathrm{D}$ convolutional layers in order to make the architecture well suitable for 3D data processing.

The model will be trained using data collected from Sharda hospital and another hospital in NCR. In training the model, End to end deep learning algorithm with the gradient-based technique will be used.

\section{CONCLUSION}

It is very clear from the discussion above that different DL models have been applied to a different task in the domain of medical imaging which has achieved high performance in detection, segmentation, classification, and registration of medical images, though they have some significant challenges and limitations. DL agonize with a poor generalization of models when used with input data that comes from different machines, while there is a lack of enough annotated medical data for training the model. Even though various techniques like transfer learning are developed to mitigate the scarcity of annotated training data, its effectiveness varies across different domain and task

Data Augmentation using Generative Adversarial Network (GAN) is proposed in this paper to overcome this limitation for better accuracy and performance. In this paper, the significance of DL in medical imaging has been surveyed and discussed. From the survey, it shows that DL has an advantage over the existing traditional machine learning with many architectures, hardware, and software frameworks. We end with a proposed model that will be used for detection, segmentation, and classification of the brain lesion.
1. H. Kasban, M. El-Bendary, and D. Salama, "A comparative study of medical imaging techniques," Int. J. Information Sci. Intelligent System, vol. 4 , pp. $37-58,2015$

2. K. Fukushima, "Neocognitron: A self-organizing neural network model for a mechanism of pattern recognition unaffected by shift in position," Biological Cybernetics, vol. 36, pp. 193-202, 1980.

3. G. E. Hinton and R. R. Salakhutdinov, "Reducing the dimensionality of data with neural networks," Science, vol. 313, pp. 504-507, 2006.

4. H. Greenspan, B. Van Ginneken, and R. M. Summers, "Guest editorial deep learning in medical imaging: Overview and future promise of an exciting new technique," IEEE Transactions on Medical Imaging, vol. 35, pp. 1153-1159, 2016

5. G. Litjens, T. Kooi, B. E. Bernardi, A. A. A. Setio, F. Ciompi, M. Ghafoorian, et al., "A survey on deep learning in medical image analysis," Medical image analysis, vol. 42, pp. 60-88, 2017.

6. B. J. Erickson, P. Korfiatis, Z. Akkus, and T. L. Kline, "Machine learning for medical imaging," Radiographics, vol. 37, pp. 505-515, 2017.

7. K. Suzuki, "Overview of deep learning in medical imaging," Radiological physics and technology, vol. 10, pp. 257-273, 2017.

8. J. Fang, A. L. Varbanescu, and H. Sips, "A comprehensive performance comparison of CUDA and OpenCL," in 2011 International Conference on Parallel Processing, 2011, pp. 216-225.

9. T. Kalaiselvi, P. Sriramakrishnan, and K. Somasundaram, "Performance analysis of morphological operations in CPU and GPU for accelerating digital image applications," International Journal of Computational Science and Information Technology (IJCSITY), pp. 15-27, 2016.

10. Y. Jia, E. Shelhamer, J. Donahue, S. Karayev, J. Long, R. Girshick, et al., "Caffe: Convolutional architecture for fast feature embedding," in Proceedings of the 22nd ACM international conference on Multimedia, 2014, pp. 675-678.

11. F. Bastien, P. Lamblin, R. Pascanu, J. Bergstra, I. Goodfellow, A. Bergeron, et al., "Theano: new features and speed improvements," arXiv preprint arXiv:1211.5590, 2012.

12. J. Bergstra, F. Bastien, O. Breuleux, P. Lamblin, R. Pascanu, O. Delalleau, et al., "Theano: Deep learning on GPUs with python," in NIPS 2011, big learning Workshop, Granada, Spain, 2011, pp. 1-48.

13. ] M. Abadi and A. A. B. P. TensorFlow, "Large-scale machine learning on heterogeneous distributed systems," in Proceedings of the 12th USENIX Symposium on Operating Systems Design and Implementation (OSDI'16) (Savannah, GA, USA, 2016, pp. 265-283.

14. R. Collobert, K. Kavukcuoglu, and C. Farabet, "Implementing neural networks efficiently," in Neural Networks: Tricks of the Trade, ed: Springer, 2012, pp. 537-557.

15. R. Collobert, K. Kavukcuoglu, and C. Farabet, "Torch7: A Matlab-like environment for machine learning," in BigLearn, NIPS workshop, 2011, p. 10.

16. R. Shokri and V. Shmatikov, "Privacy-preserving deep learning," in Proceedings of the 22nd ACM SIGSAC conference on computer and communications security, 2015, pp. 1310-1321.

17. D. Ravì, C. Wong, F. Deligianni, M. Berthelot, J. Andreu-Perez, B Lo, et al., "Deep learning for health informatics," IEEE Journal of biomedical and health informatics, vol. 21, pp. 4-21, 2017.

18. Y. LeCun, Y. Bengio, and G. Hinton, "Deep learning," Nature, vol. 521, p. 436, 2015.

19. Y. Bengio, "Learning deep architectures for AI," Foundations and Trends $₫$ in Machine Learning, vol. 2, pp. 1-127, 2009.

20. J. Schmidhuber, "Deep learning in neural networks: An overview," Neural networks, vol. 61, pp. 85-117, 2015.

21. D. Wang, A. Khosla, R. Gargeya, H. Irshad, and A. H. Beck, "Deep learning for identifying metastatic breast cancer," arXiv preprint arXiv:1606.05718, 2016.

22. M. Jermyn, J. Desroches, J. Mercier, M.-A. Tremblay, K. St-Arnaud, M.-C. Guiot, et al., "Neural networks improve brain cancer detection with Raman spectroscopy in the presence of operating room light artifacts," Journal of biomedical optics, vol. 21, p. 094002, 2016.

23. D. E. Rumelhart, G. E. Hinton, and R. J. Williams, "Learning representations by back-propagating errors," Cognitive modeling, vol. 5, p. $1,1988$. 
24. Y. LeCun and Y. Bengio, "Convolutional networks for images, speech, and time series," The handbook of brain theory and neural networks, vol. 3361, p. 1995, 1995.

25. J. Ker, L. Wang, J. Rao, and T. Lim, "Deep learning applications in medical image analysis," Ieee Access, vol. 6, pp. 9375-9389, 2018

26. S. M. Anwar, M. Majid, A. Qayyum, M. Awais, M. Alnowami, and M. K. Khan, "Medical image analysis using convolutional neural networks: a review," Journal of medical systems, vol. 42, p. 226, 2018.

27. G. E. Dahl, T. N. Sainath, and G. E. Hinton, "Improving deep neural networks for LVCSR using rectified linear units and dropout," in 2013 IEEE international conference on acoustics, speech and signal processing, 2013, pp. 8609-8613.

28. Y.-T. Zhou, R. Chellappa, A. Vaid, and B. K. Jenkins, "Image restoration using a neural network," IEEE Transactions on Acoustics, Speech, and Signal Processing, vol. 36, pp. 1141-1151, 1988.

29. T. Brosch, L. Y. Tang, Y. Yoo, D. K. Li, A. Traboulsee, and R. Tam, "Deep 3D convolutional encoder networks with shortcuts for multiscale feature integration applied to multiple sclerosis lesion segmentation," IEEE transactions on medical imaging, vol. 35, pp. 1229-1239, 2016.

30. P. Baldi, "Autoencoders, unsupervised learning, and deep architectures," in Proceedings of ICML Workshop on unsupervised and transfer learning, 2012, pp. 37-49

31. P. Vincent, H. Larochelle, I. Lajoie, Y. Bengio and P.-A. Manzagol, "Stacked denoising autoencoders: Learning useful representations in a deep network with a local denoising criterion," Journal of machine learning research, vol. 11, pp. 3371-3408, 2010.

32. Y. LeCun, L. Bottou, Y. Bengio, and P. Haffner, "Gradient-based learning applied to document recognition," Proceedings of the IEEE, vol. 86, pp. 2278-2324, 1998.

33. G. I., B. Y., and C. A., Deep Learning: MIT Press, 2016.

34. G. E. Hinton, S. Osindero, and Y.W. Teh, "A fast learning algorithm for deep belief nets," Neural Computation, vol. 18, pp. 1527-1554, 2006.

35. Y. Bengio, P. Lamblin, D. Popovici, and H. Larochelle, "Greedy Layer-Wise Training of Deep Networks," Advances in Neural Information Processing Systems, pp. 153-160, 2007.

36. R. Salakhutdinov and G. Hinton, "Deep Boltzmann Machines," in International Conference Artificial Intelligent, 2009.

37. X. Glorot, A. Bordes, and Y. Bengio, "Deep sparse rectifier neural networks," in Proceedings of the fourteenth international conference on artificial intelligence and statistics, 2011, pp. 315-323.

38. I. J. Goodfellow, J. Pouget-Abadie, M. Mirza, B. Xu, D. Warde-Farley, S. Ozair, et al., "Generative Adversarial Nets," Advances in Neural Information Processing Systems, pp. 2672-2680, 2014

39. B. J. Erickson, P. Korfiatis, T. L. Kline, Z. Akkus, K. Philbrick, and A. D. Weston, "Deep learning in radiology: does one size fit all?" Journal of the American College of Radiology, vol. 15, pp. 521-526, 2018.

40. V. Alex, M. S. KP, S. S. Chennamsetty, and G. Krishnamurthi, "Generative adversarial networks for brain lesion detection," in Medical Imaging 2017: Image Processing, 2017, p. 101330G.

41. I. Cabria and I. Gondra, "MRI segmentation fusion for brain tumor detection," Information Fusion, vol. 36, pp. 1-9, 2017.

42. D. Guo, J. Fridriksson, P. Fillmore, C. Rorden, H. Yu, K. Zheng, et al., "Automated lesion detection on MRI scans using combined unsupervised and supervised methods," BMC medical imaging, vol. 15, p. $50,2015$.

43. Y. Kobayashi, H. Kobayashi, J. T. Giles, I. Yokoe, M. Hirano, Y Nakajima, et al., "Detection of left regional ventricular dysfunction and myocardial abnormalities using complementary cardiac magnetic resonance imaging in patients with systemic sclerosis without cardiac symptoms: a pilot study," Internal Medicine, vol. 55, pp. 237-243, 2016.

44. L. Zhang and Q. Ji, "A Bayesian network model for automatic and interactive image segmentation," IEEE Transactions on Image Processing, vol. 20, pp. 2582-2593, 2011.

45. E. Smistad, T. L. Falch, M. Bozorgi, A. C. Elster, and F. Lindseth, "Medical image segmentation on GPUs-A comprehensive review," Medical image analysis, vol. 20, pp. 1-18, 2015.

46. S. Pereira, A. Pinto, V. Alves, and C. A. Silva, "Brain tumor segmentation using convolutional neural networks in MRI images," IEEE transactions on medical imaging, vol. 35, pp. 1240-1251, 2016.

47. K. Kamnitsas, E. Ferrante, S. Parisot, C. Ledig, A. V. Nori, A Criminisi, et al., "DeepMedic for brain tumor segmentation," in International workshop on Brainlesion: Glioma, multiple sclerosis, stroke and traumatic brain injuries, 2016, pp. 138-149

48. K. Kamnitsas, C. Ledig, V. F. Newcombe, J. P. Simpson, A. D. Kane, D. K. Menon, et al., "Efficient multi-scale 3D CNN with fully connected
CRF for accurate brain lesion segmentation," Medical image analysis, vol. 36, pp. 61-78, 2017.

49. M. Anthopoulos, S. Christodoulidis, L. Ebner, A. Christe, and S. Mougiakakou, "Lung pattern classification for interstitial lung diseases using a deep convolutional neural network," IEEE transactions on medical imaging, vol. 35, pp. 1207-1216, 2016.

50. K. Sirinukunwattana, S. e. A. Raza, Y.-W. Tsang, D. R. Snead, I. A Cree, and N. M. Rajpoot, "Locality sensitive deep learning for detection and classification of nuclei in routine colon cancer histology images," IEEE Trans. Med. Imaging, vol. 35, pp. 1196-1206, 2016.

51. S. Miao, Z. J. Wang, and R. Liao, "A CNN regression approach for real-time 2D/3D registration," IEEE transactions on medical imaging, vol. 35, pp. 1352-1363, 2016.

52. X. Yang, R. Kwitt, M. Styner, and M. Niethammer, "Quicksilver: Fast predictive image registration-a deep learning approach," NeuroImage, vol. 158, pp. 378-396, 2017.

53. A. Krizhevsky, I. Sutskever, and G. E. Hinton, "Imagenet classification with deep convolutional neural networks," in Advances in neural information processing systems, 2012, pp. 1097-1105.

\section{AUTHORS PROFILE}

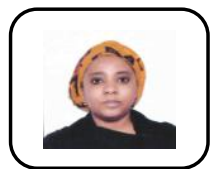

Surayya Ado Bala received the bachelor's degree in computer science from Bayero University Kano, Nigeria in 2010, the master's degree in software engineering from Sharda University, India in 2015. She is currently pursuing a Ph.D. degree with the Computer Science and Engineering Department, Sharda University, India. Her current research interests include medical image analysis, deep learning, machine learning, and data mining.

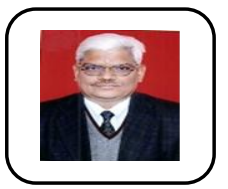

Shri Kant received Ph.D.(Mathematics) from Institute of Technology, Banaras Hindu University, India. He worked for more than 35 years in Defense Research and Development Organization (DRDO), M/O Defense in various capacities viz: Scientists, Coordinator, and Director of a DRDO lab. During this period, he has guided a team of scientists working on Pattern Recognition, Cluster Analysis, and Soft Computing application in the field of cryptology, mainly cryptanalysis. Currently, he is working as a Professor at Research and Technology Development Centre (RTDC), Dept. of Computer Science and Engineering of Sharda University, India and involved actively in teaching and research mainly in the area of cybersecurity and application of machine learning in various other areas. His areas of interest are Special Functions, Cryptology, Pattern Recognition, Cluster Analysis, and Data Mining. He has published more than eighty research papers in international and national journals and conferences and also published fifteen confidential technical reports and obtained eight copyright for different groups of algorithms developed during his career with DRDO. He has guided many PG projects and $\mathrm{Ph} . \mathrm{D}$. theses. He has received commendation certificates and scientist of the Lab award for exhibiting the excellence in pattern recognition application to cryptology.

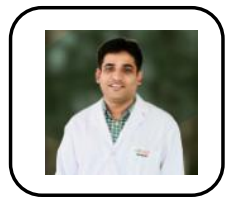

Khemendra Kumar received MBBS from BMC Pune, and he further did his MD from Dr. SNMC Jodhpur, India in 2011. Dr. Khemendra has worked at All India Institute of Medical Sciences (AIIMS), New Delhi in Department of Cardiovascular Radiology and Endovascular Interventions, and he is currently an Assistant Professor at Sharda Hospital. He has vast experience in the vita area of endovascular laser ablation of varicosities and sclerotherapies for varicose Vein management. He is also proficient in even very complicated procedures like failed surgical procedures. Dr. Khemendra has several research and publications and has participated in the Asia Pacific conference for advancing in the field of Interventional Radiology. 